\title{
Anaphylaxis to cutaneous exposure to bovine colostrum based cream
}

\author{
Federica Porcaro, ${ }^{1}$ Lucia Caminiti, ${ }^{2}$ Giuseppe Crisafulli, ${ }^{2}$ Francesco Guglielmo, ${ }^{3}$ Giovanni Battista Pajno ${ }^{2}$
}

\begin{abstract}
Children who are highly sensitive to milk may also have severe allergic reactions after exposure to cow's milk proteins (CMP) through a different administration route than the oral one. We describe the case of a 16-year-old Caucasian boy with a clinical history of persistent cow's milk allergy (CMA), who developed one episode of anaphylaxis following cutaneous application of a bovine colostrum containing cream to a surgical wound. UniCAP testing showed a significant elevation in specific IgE antibodies to whey milk proteins. Until now, only three cases of anaphylaxis following cutaneous application of products containing milk proteins were available in the scientific literature.
\end{abstract}

Keywords: anaphylaxis, asthma, bovine colostrum, cow's milk allergy, children.

\section{From:}

${ }^{1}$ Respiratory Unit, Academic Department of Pediatrics, Bambino Gesù Children's Hospital, Piazza di Sant'Onofrio, 4, 00165, Rome, Italy.

${ }^{2}$ Allergy Unit, Department of Pediatrics, University of Messina, Via

Consolare Valeria-Gazzi, 98124, Messina, Italy.

${ }^{3}$ Pediatric National Healthcare System, Catania, Italy.

\section{Introduction}

Cow's milk allergy (CMA) is the most common food allergy in pediatric patients and it affects $2-3 \%$ of children. ${ }^{1}$ https:/www.ncbi.nlm.nih.gov/pmc/articles/PMC3604421/ - R2 Cow's milk contains more than 25 different proteins, but only whey proteins $\alpha$-lactalbumin, $\beta$-lactoglobulin, bovine serum albumin (BSA), and lactoferrin as well as four caseins have been identified as allergens. ${ }^{2}$ In sensitized children, oral intake of cow's milk is responsible for $\operatorname{IgE}$ or non-IgE mediated reactions, even though skin contact and food inhalation may also trigger adverse reactions. ${ }^{3,4}$ IgE-mediated food allergy can present with skin, gastrointestinal, respiratory, and cardiovascular symptoms, and sometimes may be life threatening and treatment takes place in the emergency setting.

We describe the case of a 16-year-old boy affected by CMA and mild chronic allergic asthma with anaphylaxis to cutaneous exposure to milk proteins in a colostrum based cream.

\section{Case Report}

A Caucasian 16-year-old boy was affected by CMA since the age of 10 months. Family history was negative for atopy. He was exclusively breast-fed in the first months of life. The first adverse reaction was characterized by anaphylaxis after the ingestion of milk formula. CMA was confirmed by cutaneous and laboratory tests in the first year of life. Skin prick test (SPT) was conducted using both fresh milk and extracts of cow milk

\section{Corresponding author:}

Federica Porcaro

Respiratory Unit, Academic Department of Pediatrics, Bambino Gesù Children's Hospital, Piazza di Sant'Onofrio, 4, 00165, Rome, Italy.

Email: federica.porcaro@opbg.net

proteins including cow $\beta$-lactalbumin (Bos d 4), $\beta$-lactoglobulin (Bos d 5), and casein (Bos d 8) (Lofarma, Milan, Italy) - wheal diameters were $8 \mathrm{~mm}$ for milk, $13.5 \mathrm{~mm}$ for purified natural Bos d 4, $8 \mathrm{~mm}$ for Bos d 5, and $11 \mathrm{~mm}$ for Bos $\mathrm{d} 8$. In addition, blood samples were drawn for measurement of total IgE (597 $\mathrm{IU} / \mathrm{mL}$ ), and milk-specific IgE levels (UniCAP, Phadia, Uppsala, Sweden) were $90 \mathrm{kUA} / \mathrm{L}$ for milk, $62.3 \mathrm{kUA} / \mathrm{L}$ for Bos d 8, $62.5 \mathrm{kUA} / \mathrm{L}$ for Bos $\mathrm{d} 4$, and $36.6 \mathrm{kUA} / \mathrm{L}$ for Bos $\mathrm{d} 5$. Therefore, an oral food challenge (OFC) with cow's milk (CM) formula was undertaken and within few minutes of formula administration, the patient developed generalized hives and wheezing, confirming the diagnosis of CMA. Avoidance of milk and dairy products was recommended, use of CM hydrolysate was suggested and auto-injectable epinephrine was prescribed as emergency medication for anaphylaxis.

Despite good compliance to an elimination diet, the patient experienced two episodes of anaphylaxis after the accidental ingestion of food products containing hidden milk. Every year the patient underwent OFC to CM in order to verify the appearance of spontaneous tolerance. However, the patient showed allergic symptoms also during the last OFC to CM performed when he was 15 years old. Furthermore, asthma symptoms occurred in preschool age and were associated with sensitization to the house dust mite (SPT $4 \mathrm{~mm}$ and sIgE 1.18 kUA/L for Dermatophagoides pteronyssinus, SPT $3 \mathrm{~mm}$ and sIgE $1.12 \mathrm{kUA} / \mathrm{L}$ for Dermatophagoides farinae), dog (SPT 4 
$\mathrm{mm}$ and sIgE $1.0 \mathrm{kUA} / \mathrm{L}$ ), and cat epithelium (SPT $5 \mathrm{~mm}$ and sIgE $5.36 \mathrm{kUA} / \mathrm{L})$. Asthma control was obtained with a low dose of inhaled steroid (fluticasone propionate), and spirometry showed mild obstruction with FEV1 $73 \%$ of predicted value, FVC $96 \%$ of predicted value, and FEV1/FVC ratio of $76 \%$.

In addition, because a sacrococcygeal fistula was detected, surgical intervention was required when he was 15 years old. Six months after surgery, he applied a topical colostrum based cream (bovine colostrum, hyaluronic acid, sodium alginate, and Aloe barbadensis gel) to the surgical wound. Urticaria, itching, and wheezing occurred immediately within few minutes and symptoms disappeared after systemic epinephrine (Fastjekt $330 \mathrm{mcg}$ ), hydrocortisone (200 mg), and chlorpheniramine maleate $(10 \mathrm{mg})$ administration. The last SPT tests with cow's milk proteins (CMPs) were performed six months before the evaluation in the emergency setting and a wheal diameter of 13 $\mathrm{mm}$ for Bos d 4, $12 \mathrm{~mm}$ for Bos d 8, and $8 \mathrm{~mm}$ for Bos d 5 confirmed CM sensitization.

Because no correlation with any food ingestion was detected, the suspicion of allergic reaction due to topical application of colostrum based cream was strong. The presence of potentially allergenic milk proteins in a sample of the implicated product was assessed by immunoblotting techniques. Three bands corresponding to the $\alpha$-lactalbumin $(\alpha-\mathrm{La}), \beta$-lactoglobulin $(\beta$-Lg), and casein were detected in the bovine colostrum based cream incubated with patient sera (Figure 1).

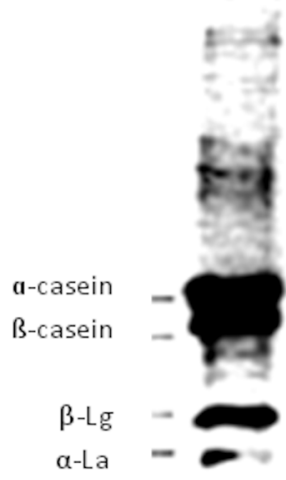

Figure 1. Immunoblotting of colostrum based cream incubated with patient sera ( $\alpha$-casein, $\beta$-casein, $\beta$-lactoglobulin, a-lactalbumin).

\section{Discussion}

IgE-mediated CMA is reported to be a major cause of anaphylaxis and mortality in children and requires compliance with strict avoidance of CM and dairy products, as well as self-carry of an epinephrine autoinjector. ${ }^{5}$ Our case draws attention to potential problematic aspects related to the management of persistent IgE-mediated food allergy. Children who are highly sensitive to milk may have severe allergic reactions not only after ingestion or cutaneous contact of culprit food but also after cutaneous or intravenous exposure to CMPs contained in some drugs or preparations.

Allergic reactions due to cutaneous allergen contact are described in children with IgE-mediated CMA and atopic dermatitis, because the skin barrier impairment facilitates the epicutaneous penetration of molecules of food allergens ${ }^{6}$ (similar reactions are also described in children with non -eczematous skin). ${ }^{7}$

Sometimes, contact with food proteins without ingestion can cause severe systemic allergic reactions. ${ }^{3,8}$ Tan et al. described five cases of children who experienced severe food allergic reactions by skin contact to milk and peanuts. Patients were characterized by strong family history of allergy, early age of onset, strong positive reactivity to foods by SPT, and multiple sensitizations to other foods. ${ }^{3}$

It is known that CMPs are present in a large variety of processed foods or drugs, but their presence may be hidden, as they are occasionally listed on the product label in a manner unrecognizable to the consumer. For this reason, the parents of more sensitive patients need to be instructed in the detection of these potential sources of hidden CMPs by carefully reading food and drug labels and in recognition of the sources; thus, avoiding possible adverse and severe reactions.

Bovine colostrum is mostly composed of $\alpha$-La (15\%-20\%) and $\beta-\operatorname{Lg}(55 \%-65 \%)$; the minor components include immunoglobulins (9\%), bovine serum albumin (5.5\%), lactoferrin, phospholipoproteins, bioactive factors, and enzymes. ${ }^{9}$ It also contains endothelial and basic fibroblast growth factors and platelet-derived growth factor. The last one is believed to be an important mitogen for fibroblasts, which explains the use of bovine colostrum in products applied for wound healing. ${ }^{10}$

As previously described, our patient was highly sensitized to specific allergen components of CMPs as shown by SPT and $\operatorname{sIgE}$ that were significantly positive for purified natural Bos d 4 of $\alpha$-La and Bos d 8 of casein. Even if he hadn't had a positive family history of atopy and other sensitizations to food, he had early-onset allergy and strong positive reactivity to CMP by SPT, so the cutaneous exposition to the culprit allergen was sufficient to induce a severe allergic reaction requiring epinephrine administration.

To the best of our knowledge, only three cases of anaphylaxis following cutaneous application of products containing CMPs are available in the scientific literature. Anaphylaxis occurred in two children after cutaneous application of a casein containing ointment to an inflamed diaper area ${ }^{11,12}$ and in an adult woman using a cosmetic that contained casein. ${ }^{13}$

\section{Conclusion}

The effective management of food allergy includes avoidance of the food allergen and instructions on the the use of epinephrine self-injection. Nevertheless, patients and their parents must be aware that other administration routes of allergen, like the cutaneous one, can cause serious reactions. Physicians must instruct patients and their parents more thoroughly about their allergies, above all specifying the relevant names of all allergens to which they are sensitized. In the future, may be the new active treatment of IgE-mediated food allergy, oral immunotherapy $(\mathrm{OIT})^{14}$ will provide a new effective therapy with the goal of increasing the threshold of reactivity and improving the quality of life of patients.

\section{Acknowledgements}

Conflict of interest: Authors have no conflicts of interest to disclose.

Source of funding with grant numbers: Not applicable. 


\section{Author contributions}

- Federica Porcaro collected data, drafted the manuscript, approved the final manuscript as submitted, and guarantees that every part of the work has been appropriately investigated and resolved.

- Lucia Caminiti contributed to data acquisition, managed the patients, critically revised the manuscript, approved the final manuscript as submitted, and guarantees that every part of the work has been appropriately investigated and resolved.

- Giuseppe Crisafulli contributed to data acquisition, managed the patients, critically revised the manuscript, approved the final manuscript as submitted, and guarantees that every part of the work has been appropriately investigated and resolved.

- Francesco Guglielmo contributed to data acquisition, managed the patients, critically revised the manuscript, approved the final manuscript as submitted, and guarantees that every part of the work has been appropriately investigated and resolved.

- Giovanni Battista Pajno coordinated and supervised data collection, critically revised the manuscript, approved the final manuscript as submitted, and guarantees that every part of the work has been appropriately investigated and resolved.

\section{References}

1. Sicherer SH, Sampson HA. Food allergy: epidemiology, pathogenesis, diagnosis, and treatment. J Allergy Clin Immunol. 2014;133:291-307.

2. Wal JM. Cow's milk allergens. Allergy. 1998;53:1013-22.

3. Tan BM, Sher MR, Good RA, Bahna SL. Severe food allergies by skin contact. Ann Allergy Asthma Immunol. 2001;86:583-6.

4. Vitaliti G, Morselli I, Di Stefano V, Lanzafame A, La Rosa M, Leonardi S. Urticaria and anaphilaxis in a child after inhalation of lentils vapours: a case report and literature review. Ital J Pediatr. 2012;38:71.

5. Worm M, Eckermann O, Dölle S, Aberer W, Beyer K, Hawranek T et al. Triggers and Treatment of Anaphylaxis: An Analysis of 4000 Cases From Germany, Austria and Switzerland. Dtsch Arztebl Int. 2014;111:367-75.

6. Schichter-Konfino V, Almog M, Bamberger E, Berkowitz D, Kessel A. The significance of allergic contact urticaria to milk in children with cow's milk allergy. Pediatr Allergy Immunol. 2015;26:218-22.

7. Wüthrich B. Food-induced cutaneous adverse reactions. Allergy. 1998;53: 131-5.

8. Liccardi G, De Falco F, Gilder JA, D'Amato M, D’Amato G. Severe systemic allergic reaction induced by accidental skin contact with cow milk in a 16-year-old boy. A case report. J Investig Allergol Clin Immunol. 2004;14:168-71.

9. Kim J, Paik HD, Yoon YC, Park E. Whey protein inhibits iron overload -induced oxidative stress in rats. J Nutr Sci Vitamino. 2013;59:198-205.

10. Sacerdote P, Mussano F, Franchi S, Panerai AE, Bussolati G, Carossa S, et al. Biological components in a standardized derivative of bovine colostrum. J Dairy Sci. 2013;96:1745-1754.

11. Jarmoc LM, Primack WA. Anaphylaxis to cutaneous exposure to milk protein in a diaper rash ointment. Clin Pediatr. 1987;26:154-5.

12. Lecks HI. Anaphylaxis from milk protein in diaper ointment. JAMA. 1980;244:1560.

13. Tabar AI, Alvarez MJ, Echechipia S, Acero S, Garcia BE, Olaguibel JM. Anaphylaxis from cow's milk casein. Allergy. 1996;51:343-5.

14. Nurmatov U, Dhami S, Arasi S, Pajno GB, Fernandez-Rivas M, Muraro A, et al. Allergen immunotherapy for IgE-mediated food allergy: a systematic review and meta-analysis. Allergy. 2017;72:1133-1147. 